\title{
Combination of gut microbiota and plasma amyloid- $\beta$ as a potential index for identifying preclinical Alzheimer's disease: a cross-sectional analysis from the SILCODE study
}

\author{
Can Sheng ${ }^{1}$, Kun Yang ${ }^{2,3}$, Beiqi He ${ }^{4}$, Wenying Du ${ }^{1}$, Yanning Cai ${ }^{5,6,7}$ and Ying Han ${ }^{1,4,8,9^{*}}$ (D)
}

\begin{abstract}
Background: Plasma amyloid- $\beta$ (AB) may facilitate identification of individuals with brain amyloidosis. Gut microbial dysbiosis in Alzheimer's disease (AD) is increasingly being recognized. However, knowledge about alterations of gut microbiota in preclinical $A D$, as well as whether the combination of plasma $A \beta$ and gut microbiota could identify preclinical $A D$, remains largely unknown.
\end{abstract}

Methods: This study recruited $34 \mathrm{~A} \beta$-negative cognitively normal (CN-) participants, $32 \mathrm{~A} \beta$-positive cognitively normal $(\mathrm{CN}+)$ participants, and 22 patients with cognitive impairment $(\mathrm{Cl})$, including 11 patients with mild cognitive impairment $(\mathrm{MCl})$ and $11 \mathrm{AD}$ dementia patients. All participants underwent neuropsychological assessments and fecal microbiota analysis through 165 ribosomal RNA (rRNA) Illumina Miseq sequencing technique. Meso Scale Discovery (MSD) kits were used to quantify the plasma $A \beta_{40}, A \beta_{42}$, and $A \beta_{42} / A \beta_{40}$ in $C N-$ and $C N+$ participants. Using Spearman's correlation analysis, the associations of global standard uptake value rate (SUVR) with altered gut microbiota and plasma $A \beta$ markers were separately evaluated. Furthermore, the discriminative power of the combination of gut microbiota and plasma $A \beta$ markers for identifying $C N+$ individuals was investigated.

Results: Compared with the $C N-$ group, the $C N+$ group showed significantly reduced plasma $A \beta_{42}(p=0.011)$ and $A \beta_{42} / A \beta_{40}(p=0.003)$. The relative abundance of phylum Bacteroidetes was significantly enriched, whereas phylum Firmicutes and class Deltaproteobacteria were significantly decreased in $\mathrm{CN}+$ individuals in comparison with that in $\mathrm{CN}$ - individuals. Particularly, the relative abundance of phylum Firmicutes and its corresponding SCFA-producing bacteria exhibited a progressive decline tendency from $\mathrm{CN}-$ to $\mathrm{CN}+$ and $\mathrm{Cl}$. Besides, the global brain $\mathrm{A} \beta$ burden was negatively associated with the plasma $A \beta_{42} / A \beta_{40}(r=-0.298, p=0.015)$, family Desulfovibrionaceae $(r=-0.331, p=$ 0.007), genus Bilophila ( $r=-0.247, p=0.046)$, and genus Faecalibacterium $(r=-0.291, p=0.018)$ for all CN participants. Finally, the combination of plasma $A \beta$ markers, altered gut microbiota, and cognitive performance reached a relatively good discriminative power in identifying individuals with $\mathrm{CN}+$ from $\mathrm{CN}-(\mathrm{AUC}=0.869,95 \% \mathrm{Cl} 0.782 \sim$ 0.955).

\footnotetext{
*Correspondence: hanying@xwh.ccmu.edu.cn

${ }^{9}$ National Clinical Research Center for Geriatric Diseases, Beijing 100053,

China
}

Full list of author information is available at the end of the article

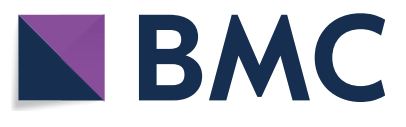

(c) The Author(s) 2022. Open Access This article is licensed under a Creative Commons Attribution 4.0 International License, which permits use, sharing, adaptation, distribution and reproduction in any medium or format, as long as you give appropriate credit to the original author(s) and the source, provide a link to the Creative Commons licence, and indicate if changes were made. The images or other third party material in this article are included in the article's Creative Commons licence, unless indicated otherwise in a credit line to the material. If material is not included in the article's Creative Commons licence and your intended use is not permitted by statutory regulation or exceeds the permitted use, you will need to obtain permission directly from the copyright holder. To view a copy of this licence, visit http://creativecommons.org/licenses/by/4.0/. The Creative Commons Public Domain Dedication waiver (http://creativeco mmons.org/publicdomain/zero/1.0/) applies to the data made available in this article, unless otherwise stated in a credit line to the data. 
Conclusions: This study provided the evidence that the gut microbial composition was altered in preclinical AD. The combination of plasma $A \beta$ and gut microbiota may serve as a non-invasive, cost-effective diagnostic tool for early $A D$ screening. Targeting the gut microbiota may be a novel therapeutic strategy for AD.

Trial registration: This study has been registered in ClinicalTrials.gov (NCT03370744, https://www.clinicaltrials.gov) in November 15, 2017.

Keywords: Alzheimer's disease, Preclinical, Gut microbiota, Amyloid- $\beta$, Plasma

\section{Introduction}

Alzheimer's disease (AD) is a progressive and irreversible neurodegenerative disorder, causing globally heavy healthcare burden [1]. Increasing evidence implicates that the pathophysiological process of AD begins 15-20 years before the emergence of clinical symptoms [2, 3]. Given the lack of effective strategies available for delaying or preventing the progression of $\mathrm{AD}$, interventions targeting the preclinical stage of $\mathrm{AD}$ may offer the best chance for therapeutic success $[2,4]$. Currently, preclinical $\mathrm{AD}$ is defined by biomarker evidence of AD-related pathological changes in cognitively healthy individuals. Abnormal amyloid positron emission tomography (PET) scan or low cerebrospinal fluid (CSF) amyloid- $\beta(\mathrm{A} \beta)_{42}$ or $A \beta_{42} / A \beta_{40}$ ratio are considered as the evidence of $A \beta$ deposition. It is noteworthy that although amyloid PET is gaining attraction in clinical practice, expensive costs and radioactivity limit its wide application. In addition, lumbar puncture is an invasive procedure, and repeated CSF collection is also challenging. Therefore, exploring novel and potentially alternative hallmarks for identifying preclinical AD are needed.

Gut microbiota is considered as a possible susceptibility factor for $\mathrm{AD}$ [5]. Accumulating findings support that gut microbiota has the potential to modulate brain function, such as memory and learning [6, 7]. Cross-sectional preclinical and clinical studies provide the insight that altered gut microbial compositions may contribute to the AD pathology $[5,8]$, and manipulating gut microbiota can attenuate brain $A \beta$ deposition $[9,10]$. Previous studies have demonstrated significant alterations of gut microbiota in patients with $\mathrm{AD}$ and $\mathrm{MCI}$ compared with healthy controls, such as decreased phylum Firmicutes, increased family Enterobacteriaceae [11-13]. One recent study also reported the decreased anti-inflammatory genus Faecalibacterium in individuals with subjective cognitive decline $(\mathrm{SCD})$, providing the preliminary evidence of altered gut microbiota in elderly adults at risk of $\mathrm{AD}$ [14]. However, there still exist some issues regarding current $\mathrm{AD}$ microbiome studies. Firstly, it is still unclear whether similar alterations of gut microbial compositions occur in the stage of preclinical $\mathrm{AD}$ in vivo. Secondly, in previous researches, the diagnosis of $\mathrm{AD}$ and MCI patients was based on clinical symptoms and lack of pathophysiological biomarkers. Therefore, in this study, we aimed to investigate the characteristics of gut microbiota in asymptomatic preclinical individuals with biomarker evidence of $A \beta$ deposition.

Moreover, the peripheral blood may be another promising source for screening AD biomarkers. Previous studies have reported the association of changed plasma $A \beta$ with $\mathrm{AD}$ [15-18]. Plasma $A \beta$ seems to be a potential hallmark detecting brain $A \beta$ pathological changes [16, 19, $20]$. A recent study showed that plasma $A \beta_{42 / 40}$ ratio had the potential to identify brain $A \beta$ positivity in preclinical $A D$ individuals $[20]$, suggesting that plasma $A \beta$ may be used as a diagnostic tool in routine clinical work. However, previous published studies regarding the correlation between plasma $A \beta$ and $A D$ pathology are contradictory, especially for plasma $A \beta_{40}$ and $A \beta_{42}$.

The main purposes of this study were (1) to characterize the gut microbiota in the preclinical stage of $A D,(2)$ to assess whether plasma $A \beta$ indexes $\left(A \beta_{40}, A \beta_{42}\right.$, and the ratio of $A \beta_{40}$ and $A \beta_{42}$ ) were changed in preclinical $\mathrm{AD}$, and (3) to investigate the discriminative power of the combined gut microbiota and plasma $A \beta$ indexes in identifying individuals with preclinical AD.

\section{Materials and methods \\ Participants}

In the present study, we recruited a total of 66 righthanded Chinese participants, including $34 \mathrm{~A} \beta$-negative cognitively normal $(\mathrm{CN}-)$ participants and 32 A $\beta$-positive cognitively normal $(\mathrm{CN}+)$ participants from the Sino Longitudinal Study on Cognitive Decline (SILCODE) [21]. Each participant underwent routine clinical evaluation, standardized neuropsychological assessments, blood sample tests, fecal sample amplicon sequencing, and A $\beta$-PET scans. To eliminate the potential influence of different lifestyles (e.g., diet, exercise), ethnicities, and regions on gut microbial compositions, all participants recruited in our study were communitydwelling Han nationality older adults who resided in Beijing for a long time. In addition, each participant finished a semi-structured interview to evaluate their lifestyles in detail (Supplementary Table S1). Participants were diagnosed as $\mathrm{CN}$ - according to the following criteria: (1) normal performance on a battery of neuropsychological 
tests, (2) with negative $A \beta$ deposition in amyloid PET, and (3) failure to meet the criteria for $\mathrm{MCI}$ and dementia. Participants were defined as $\mathrm{CN}+$ (preclinical AD) when they matched the criteria: (1) normal performance on standardized neuropsychological tests, (2) aggregated $\mathrm{A} \beta$ evidence derived from amyloid PET, and (3) failure to meet the criteria for MCI and dementia. The software G*Power 3.1 was used to estimate the sample size in our study (Supplementary Methods).

We also collected the clinical and fecal data of $11 \mathrm{MCI}$ and $11 \mathrm{AD}$ patients from the SILCODE. In our study, patients with $\mathrm{MCI}$ and $\mathrm{AD}$ were defined as individuals with cognitive impairment (CI). The definition of MCI was in accordance with the criteria proposed by Jak and Bondi in 2014 [22], which met any one of the following three conditions and failed to meet the criteria for dementia: (1) having impaired scores (defined as $>1$ SD below the age/education-corrected normative means) on both measures in at least one cognitive domain (memory, language, or speed/executive function); (2) having impaired scores in each of the three cognitive domains (memory, language, or speed/executive function); and (3) the Functional Activities Questionnaire (FAQ) $\geq 9$. Patients with AD dementia were diagnosed according to the Diagnostic and Statistical Manual of Mental Disorders (fifth edition), and the guidelines for dementia due to AD proposed by the National Institute on Aging and Alzheimer's Association (NIA-AA) workgroups [23]. The diagnosis of CI patients was mainly based on clinical symptoms, and their pathophysiological features were not confirmed by amyloid PET or CSF markers in this study.

The exclusion criteria included (1) a history of stroke; (2) major depression, with Hamilton Depression Rating Scale (HAMD) score $>24$ points; (3) other central nervous system diseases that may cause cognitive impairment, such as Parkinson's disease, tumors, encephalitis and epilepsy; (4) traumatic brain injury; (5) systemic diseases, such as thyroid dysfunction, syphilis and HIV; (6) psychosis or congenital mental developmental delay; (7) a history of using antibiotics, probiotics, prebiotics, or synbiotics within 3 months before fecal sample collection; (8) the use of corticosteroid, immune stimulating medications, and immunosuppressive agents; (9) major gastrointestinal tract surgery in past 5 years; and (10) severe gastrointestinal diseases, such as irritable bowel syndrome, inflammatory bowel disease, severe gastritis, other dysfunction in digestion and absorption, which has been reported to influence gut microbiota.

This study was registered on ClinicalTrials.gov (Identifier: NCT03370744), and research activities were approved by the Medical Research Ethics Committee and Institutional Review Board of Xuanwu Hospital in the Capital Medical University (ID: [2017]046). Each participant needed to provide a written informed consent before participating in study procedures.

\section{Clinical data collection and neuropsychological assessments}

Clinical data, including age, sex, years of education, body mass index (BMI), apolipoprotein E (APOE) genotype, and medical history of hypertension and diabetes, were collected. All participants carried on a battery of standardized neuropsychological tests as follows: (1) memory domain: the Auditory Verbal Learning Test-HuaShan version [AVLT-H] [24], including AVLT-long delayed recall and AVLT-recognition; (2) executive domain: the Shape Trails Test Part A (STT-A) and the Shape Trails Test Part B (STT-B) [25]; (3) language domain: the Animal Fluency Test (AFT) [26] and the 30-item Boston Naming Test (BNT) [27]; (4) global cognitive function: the Montreal Cognitive Assessment-Basic Version (MoCA-B) [28]; (5) daily functional activities: the FAQ; and (6) mood status: the Hamilton Depression Rating Scale (HAMD) and the Hamilton Anxiety Rating Scale (HAMA).

\section{Fecal sample collection and DNA extraction}

Participants were asked to collect a fresh fecal sample in the morning using certain fecal collection containers (SARSTEDT, Germany). All the samples were transferred to the laboratory and stored at $-80^{\circ} \mathrm{C}$ prior to processing. The DNA in each fecal sample was extracted using a QIAamp DNA Stool Mini Kit (Qiagen, Hilden, Germany). The procedures of DNA extraction were conducted under a Class II biologic safety cabinet. Then, the Thermo NanoDrop 2000 spectrophotometer (Thermo Scientific, MA, USA) was used to quantify the concentration of genomic DNA. The DNA integrity and fragment sizes were assessed using 1\% agarose gel electrophoresis (AGE). After that, DNA was re-stored at $-80^{\circ} \mathrm{C}$ prior to subsequent analysis.

\section{S rRNA gene amplicon sequencing}

The amplicon sequencing procedures were performed in an Illumina Miseq PE250 platform [29]. The V3-V4 region of the bacterial $16 \mathrm{~S}$ ribosomal RNA (rRNA) gene was selected for the amplification. There were two universal primers linking with indices and sequencing adaptors. The forward primer $\left(5^{\prime}-3^{\prime}\right)$ was CCTACGGGRSGCA GCAG $(341 \mathrm{~F})$, and the reverse primer $\left(5^{\prime}-3^{\prime}\right)$ was GGA CTACVVGGGTATCTAATC (806R). Using a KAPA HiFi Hotstart ReadyMix polymerase chain reaction (PCR) kit, the genomic DNA was utilized as a template for PCR amplification. The PCR products were examined using 2\% AGE, and gel extraction was conducted by AxyPrep DNA Gel Extraction Kit (Axygen Biosciences, 
Union City, CA, USA). Subsequently, the concentration of DNA was quantified by the Thermo NanoDrop 2000 spectrophotometer (Thermo Scientific, MA, USA), and the quantity of DNA was assessed using 2\% AGE. Finally, sequencing libraries were quantified using Qubit and then pooled to obtain a sufficient concentration.

\section{Sequence analysis}

The sequence analysis procedures were conducted according to our previous study [14]. Paired-end reads were concatenated into longer tags based on the $3^{\prime}$ overlapping regions by VSEARCH (https://github.com/torog nes/vsearch). VSEARCH is an open source multithreaded 64-bit tool for processing and preparing amplicon analysis [30]. Then, the primers of merged reads were cut and quality filter was conducted to keep reads error rates less than $1 \%$. After the dereplication, denoised sequences called "zero-radius operational taxonomic units" (ZOTUs) were generated using USEARCH 10 (http:// www.drive5.com/usearch/) [31]. Denoising was done by the unoise3 command (http://www.drive5.com/usearch/ manual/unoise_algo.html), which was used to identify all correct biological sequences in the reads. Taxonomy was assigned using the Ribosomal Database Project (RDP) as the reference database. After generating the amplicon sequence variants (ASV) table, all samples were normalized to the same number of reads.

Additionally, alpha diversity and beta diversity indexes were calculated based on normalized ASV counts using the online analysis (MicrobiomeAnalyst, https://www. microbiomeanalyst.ca/MicrobiomeAnalyst/home.xhtml). Alpha diversity means the diversity in a single ecosystem or sample. The main metrics of alpha diversity included Chao1, ACE, Shannon, and Simpson in our study. The Chao 1 and ACE metrics were used to evaluate the number of ZOTU, which mainly reflected the community richness in a sample. The Channon and Simpson metrics focusing on assessing the community diversity of a sample. Beta diversity were employed to exhibit the different gut microbial communities between different groups. Core microbiota based on the ASV level was also identified by Core microbiome analysis (sample prevalence $=$ $20 \%$, relative abundance $=0.01 \%$ ) in MicrobiomeAnalyst.

\section{Plasma $A \beta$ tests}

Blood samples (2-ml venous blood) were collected between 7: 00 and 8: 00 in the morning after an overnight fast using EDTA tubes. After repeated centrifugation for $15 \mathrm{~min}$ at $4^{\circ} \mathrm{C}$ (speed: $2500 \mathrm{~g} / \mathrm{min}$ ), supernatants were collected as the plasma. All plasma samples were stored at $-80^{\circ} \mathrm{C}$ and thawed immediately on ice before assaying. In our study, the concentration of plasma $A \beta_{40}$ and $\mathrm{A} \beta_{42}$ was quantified using Meso Scale Discovery (MSD) method. V-PLEX A $\beta$ Peptide Panel 1 (4GB): K15199e kits (MSD, Rockville, Maryland, USA) were used. All samples were measured in duplicate using the same aliquot following the manufacturer's instructions. The interand intra-plate coefficient of variation for plasma $A \beta_{40}$ and $A \beta_{42}$ was within $5 \%$. Plasma $A \beta_{40}, A \beta_{42}$, and their ratio $\left(A \beta_{42} / A \beta_{40}\right)$ indexes were used for the subsequent analysis.

\section{Neuroimaging data acquisition}

In SILCODE, $\left[{ }^{18} \mathrm{~F}\right]$ florbetapir (AV-45) PET and MRI scans were performed on an integrated simultaneous 3.0 T TOF PET/MR scanner (SIGNA PET/MR, GE Healthcare, Milwaukee, Wisconsin, USA) at Xuanwu Hospital of Capital Medical University, Beijing. After an intravenous injection of $7-10 \mathrm{mCi}\left[{ }^{18} \mathrm{~F}\right]$ florbetapir radiotracer, participants had a rest for approximately $40 \mathrm{~min}$. Then, a 20-min static PET scan was acquired. The PET data were obtained using a time-of-flight ordered subset expectation maximization (TOF-OSEM) algorithm with the following parameters: 8 iterations, 32 subsets matrix $=192$ $\times 192$, field of view $(F O V)=350 \times 350 \mathrm{~mm}^{2}$, and halfwidth height $=3$. The parameters for T1-weighted $3 \mathrm{D}$ brain structural images were as follows: SPGR sequence, FOV $=256 \times 256 \mathrm{~mm}^{2}$, matrix $=256 \times 256$, slice thickness $=1 \mathrm{~mm}$, gap $=0$, slice number $=192$, repetition time $(\mathrm{TR})=6.9 \mathrm{~ms}$, echo time $(\mathrm{TE})=2.98 \mathrm{~ms}$, inversion time $(\mathrm{TI})=450 \mathrm{~ms}$, flip angle $=12^{\circ}$, and voxel size $=$ $1 \times 1 \times 1 \mathrm{~mm}^{3}$.

\section{Imaging preprocessing and analysis}

The $\left[{ }^{18} \mathrm{~F}\right]$ florbetapir PET images were preprocessed using the Statistical Parametric Mapping (SPM12) toolbox (http://www.fil.ion.ucl.ac.uk/spm/software/spm12/). PET images were registered to the corresponding $\mathrm{T} 1$ images, which were then segmented into GM, white matter, and CSF tissue probability maps. Furthermore, the registered PET images were nonlinearly registered into the Montreal Neurological Institute (MNI) stereotactic template and resampled into $3 \times 3 \times 3 \mathrm{~mm}^{3}$ voxels. Finally, normalized PET images were smoothed by a Gaussian isotropic kernel with an $8 \mathrm{~mm}$ full-width at half maximum (FWHM) to improve the signal-to-noise ratio. Global standard uptake value rate (SUVR) of PET scan was calculated as an average of SUVs in the whole brain with the cerebellum as the reference region [32]. Positive $A \beta$ burden was defined when the SUVR was above or equal to 1.18 based on the previous studies [32, 33].

\section{Statistical analysis}

The IBM SPSS Statistics 26.0 and R-3.6.3 were used for the statistical analysis. A Shapiro-Wilk test was used to confirm data normality. Demographic information, 
neuropsychological assessments, and plasma $A \beta$ indexes were compared using the two-sample $t$ test, Mann-Whitney $U$ test or Pearson's chi-squared test as appropriate. Venn diagram was drawn with the R package "VennDiagram". Mann-Whitney $U$ test was performed to compare alpha diversity indexes between the $\mathrm{CN}-$ and $\mathrm{CN}+$ group, while Kruskal-Wallis test was used to compare the alpha diversity among the $\mathrm{CN}-$, $\mathrm{CN}+$, and $\mathrm{CI}$ groups. Beta diversity was calculated using the principal coordinate analysis (PCoA) and permutational multivariate analysis of variance (PERMANOVA) based on Bray-Curtis index. We also used non-metric multidimensional scaling (NMDS) and analysis of similarities (ANOSIM) to calculate the statistical significance. Linear discriminant analysis (LDA) effect size (LEfSe) method (http://huttenhower.sph. harvard.edu/lefse/) was used to identify differentially abundant taxa between the $\mathrm{CN}+$ and $\mathrm{CN}-$ group, with an alpha cutoff of 0.05 and an effect size cutoff of 2.0. The general linear models (GLMs) were further employed to evaluate the differences of these gut microbiota identified by the LEfSe, with age, sex, BMI, and APOE as possible confounding factors. In addition, for taxa with a prevalence $\geq 1 \%$, we also evaluated taxonomic differences at the phylum, class, order, family, and genus levels using the Mann-Whitney $U$ test, with Bonferroni adjustment. Taxonomic differences among $\mathrm{CN}-, \mathrm{CN}+$, and $\mathrm{CI}$ was calculated using the KruskalWallis test. The associations of global brain SUVR with altered gut microbiota and plasma $A \beta$ markers were separately evaluated using Spearman's correlation analysis.

To determine whether the combination of altered gut microbiota and plasma $A \beta$ have the potential to distinguish individuals with $\mathrm{CN}+$ from $\mathrm{CN}-$, receiver operating characteristic (ROC) curve and the area under the ROC curve (AUC) were calculated. Multivariable logistic regression models based on gut microbiota with significant group differences and plasma $A \beta$ indexes were separately built in a stepwise manner. The ROC curves were compared with Delong's statistic method using MedCalc19.0.4 software [34]. Statistical significance was set as $p<0.05$.

\section{Results}

\section{Demographic information, neuropsychological assessments, and plasma $A \beta$}

The detailed demographics of all $\mathrm{CN}$ participants in this study can be found in Table 1. No significant differences were found in age, sex, years of education, BMI, APOE $\varepsilon 4$ carrier, diabetes, hypertension, and emotional status between the two groups (all $p>0.05$ ). The $\mathrm{CN}+$ group showed significantly lower score in the AVLT-long
Table 1 Demographics and neuropsychological assessments for all CN participants

\begin{tabular}{|c|c|c|c|}
\hline & $\mathrm{CN}-(n=34)$ & $\mathrm{CN}+(n=32)$ & $P$ value \\
\hline \multicolumn{4}{|c|}{ Demographic information } \\
\hline Age (years) & $66.91 \pm 5.28$ & $68.44 \pm 5.35$ & 0.507 \\
\hline $\operatorname{Sex}(M / F)$ & $8 / 26$ & $10 / 22$ & 0.482 \\
\hline Education (years) & $12.76 \pm 2.99$ & $12.69 \pm 2.62$ & 0.901 \\
\hline $\mathrm{BMI}$ & $23.78 \pm 2.90$ & $24.42 \pm 2.74$ & 0.287 \\
\hline APOE $\varepsilon 4(\%)$ & $11(32.35 \%)$ & $12(37.50 \%)$ & 0.661 \\
\hline Diabetes (\%) & $5(14.71 \%)$ & $1(3.13 \%)$ & 0.102 \\
\hline Hypertension (\%) & 15 (44.12\%) & $12(37.50 \%)$ & 0.585 \\
\hline \multicolumn{4}{|c|}{ Neuropsychological tests } \\
\hline HAMD & $2.88 \pm 3.51$ & $3.81 \pm 3.83$ & 0.199 \\
\hline HAMA & $3.44 \pm 3.26$ & $3.75 \pm 2.65$ & 0.407 \\
\hline MoCA-B & $25.76 \pm 2.73$ & $26.28 \pm 2.76$ & 0.385 \\
\hline AVLT-D (long) & $8.74 \pm 1.66$ & $7.75 \pm 1.88$ & $0.018^{*}$ \\
\hline AVLT-R & $22.94 \pm 1.13$ & $22.63 \pm 1.62$ & 0.489 \\
\hline STT-A & $51.47 \pm 15.72$ & $62.53 \pm 24.66$ & 0.088 \\
\hline STT-B & $121.09 \pm 32.60$ & $142.22 \pm 47.95$ & $0.039^{*}$ \\
\hline AFT & $18.74 \pm 4.59$ & $19.91 \pm 3.95$ & 0.272 \\
\hline BNT & $25.65 \pm 2.86$ & $26.25 \pm 2.66$ & 0.342 \\
\hline FAQ & $1.00 \pm 1.72$ & $0.97 \pm 1.12$ & 0.333 \\
\hline
\end{tabular}

Abbreviations: $\mathrm{CN}$ - amyloid- $\beta$-negative cognitively normal participants, $\mathrm{CN}+$ amyloid- $\beta$-positive cognitively normal participants, $M$ male, $F$ female, $B M I$ body mass index, APOE apolipoprotein E, HAMD Hamilton Depression Rating Scale, HAMA Hamilton Anxiety Rating Scale, MoCA-B Montreal Cognitive AssessmentBasic version, AVLT-D (long) Auditory Verbal Learning Test-long delayed recall, AVLT-R Auditory Verbal Learning Test-recognition, STT-A Shape Trails Test Part A, STT-B Shape Trails Test Part B, AFT Animal Fluency Test, BNT Boston Naming Test, $F A Q$ Functional Activities Questionnaire

${ }^{*} \mathrm{P}<0.05$, comparison between $\mathrm{CN}-$ and $\mathrm{CN}+$

delayed recall $(p=0.018)$ and higher score in the STT-B $(p=0.039)$ than that in the $\mathrm{CN}-$ group. However, there was no significant difference between the $\mathrm{CN}-$ and $\mathrm{CN}+$ groups in other neuropsychological tests, including MoCA-B, AVLT-recognition, STT-A, AFT, BNT, and FAQ $(p>0.05)$. In addition, the characteristics of $\mathrm{CI}$ patients $(n=22, \mathrm{MCI}=11, \mathrm{AD}=11)$ are shown in Supplementary Table S2.

Compared with the $\mathrm{CN}-$ group, the $\mathrm{CN}+$ group exhibited significantly reduced plasma $\mathrm{A} \beta_{42}(\mathrm{CN}-$ vs. $\mathrm{CN}+: 12.97 \pm 5.63$ vs. $9.84 \pm 3.62, p=0.011)$ and $\mathrm{A} \beta_{42} /$ $\mathrm{A} \beta_{40}(\mathrm{CN}-$ vs. $\mathrm{CN}+: 0.018 \pm 0.007$ vs. $0.013 \pm 0.004$, $p=0.003)$, but no significant difference in plasma $\mathrm{A} \beta_{40}$ $(\mathrm{CN}-$ vs. $\mathrm{CN}+: \mathrm{721.98} \pm 141.44$ vs. $750.48 \pm 133.91, p=$ 0.461) (Fig. 1).

\section{The gut microbiota community profiling}

As shown in a Venn diagram, the total abundance of ASVs was 2173, and 1929 ASVs were shared in both groups (Fig. 2A). Noteworthy, 147 ASVs were unique to the $\mathrm{CN}+$ group. There were 16 key ASVs between 

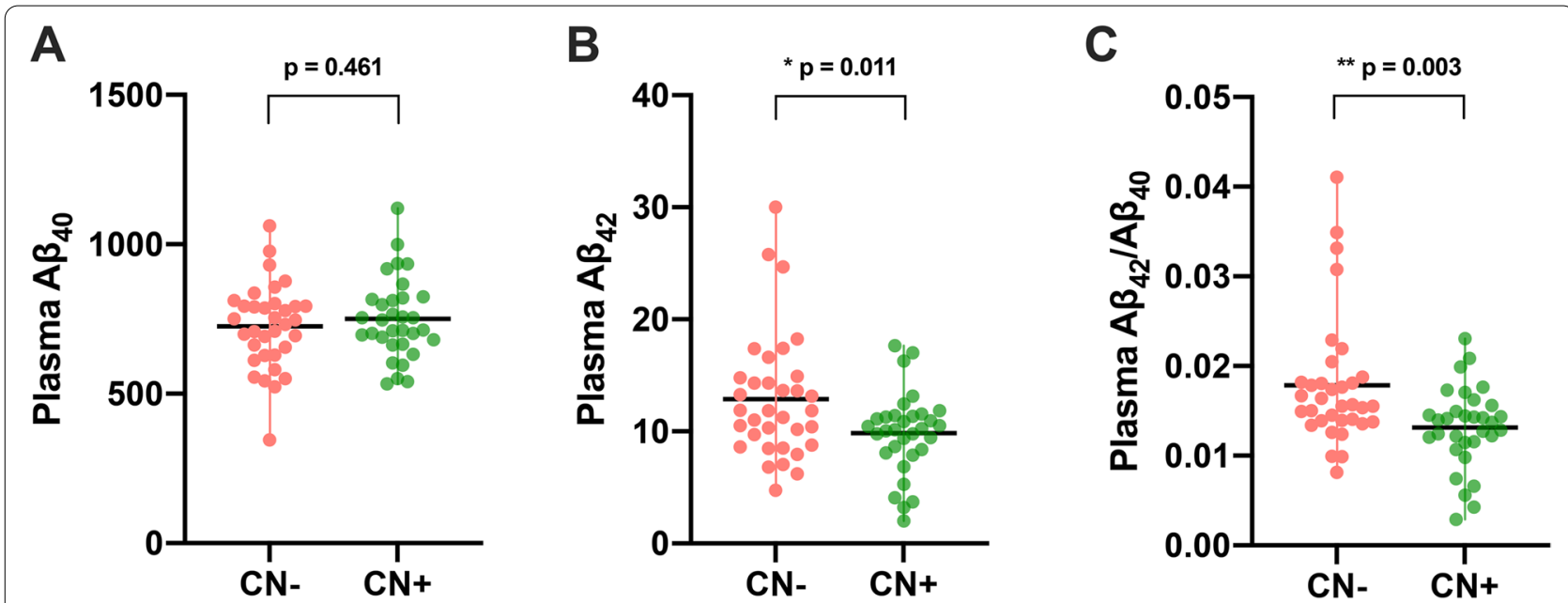

Fig. 1 Plasma $A \beta$ levels for $\mathrm{CN}$ - and $\mathrm{CN}+$ participants. Scatter plot presented mean with range. No significant difference was observed in plasma $A \beta_{40}(\mathbf{A})$, whereas there were significant differences in plasma $A \beta_{42}(\mathbf{B})$ and $A \beta_{42} / A \beta_{40}(\mathbf{C})$ between $C N-$ and $C N+.{ }^{*} p<0.05 ;{ }^{* *} p<0.01$. $A \beta$ amyloid- $\beta$; $C N$-, amyloid- $\beta$-negative cognitively normal participants; $C N+$, amyloid- $\beta$-positive cognitively normal participants

the two groups, and the abundance of two ASVs were significantly lower in the $\mathrm{CN}+$ group than in the $\mathrm{CN}-$ group (ASV_19: $p=0.0051$; ASV_9: $p=0.025)$ (Fig. 2B). Although the four alpha diversity indexes of the $\mathrm{CN}+$ group exhibited a decreased tendency relative to the $\mathrm{CN}$ - group, no statistically significant differences were found between the two groups (all $p>0.05$ ) (Fig. 2C). The PCoA based on the distribution of ASVs were conducted to illustrate the microbiome space of different samples. However, we found no significant differences in the composition of gut microbiota between the $\mathrm{CN}-$ and $\mathrm{CN}+$ groups (PERMANOVA, Bray-Curtis: $F=1.229, p<$ 0.128) (Fig. 2D).

We further investigated the alpha diversity and beta diversity among the $\mathrm{CN}-, \mathrm{CN}+$, and $\mathrm{CI}$ groups. As shown in Figure S1, there was a trend towards increasingly decrease in the Chao1, ACE, and Shannon indexes from $\mathrm{CN}-$ to $\mathrm{CN}+$ and $\mathrm{CI}$. Compared with the $\mathrm{CN}-$ group, the $\mathrm{CI}$ group showed significant decline in the Chaol $(p=0.023)$ and ACE indexes $(p=0.021)$ (Figure S1A, S1B). Moreover, the NMDS based on ASV distribution showed that the gut taxonomic composition was significantly different between $\mathrm{CN}-$ and $\mathrm{CI}$ $(R=0.097, p<0.009)$, and the PCoA showed that the difference between the two groups was marginal in statistical significance $(F=1.383, p<0.052)$ (Figure S2A, $\mathrm{S} 2 \mathrm{~B})$, suggesting that the fecal microbial structure in CI was significantly different from that of $\mathrm{CN}-$. However, for the beta diversity between $\mathrm{CN}+$ and $\mathrm{CI}$, the PCoA and the NMDS showed no gut taxonomic differences (Figure S2C, S2D).

\section{The alteration of gut microbial compositions in $\mathrm{CN}+$ participants}

The overall gut microbial compositions of the $\mathrm{CN}-$ and $\mathrm{CN}+$ groups are shown in Fig. 3A at different taxonomic levels. At the phylum level, the predominant bacteria in each group were Bacteroidetes, Firmicutes, and Proteobacteria, followed by Actinobacteria. At the class level, Bacteroidia, Clostridia, Negativicutes, Gammaproteobacteria, and Betaproteobacteria were dominant bacteria. At the order level, there were five dominant bacteria, including Bacteroidales, Clostridiales, Selenomonadales, Enterobacteriales, and Burkholderiales. At the family level, the dominant bacteria included Bacteroidaceae, Lachnospiraceae, Ruminococcaceae, and Prevotellacea. Above all, the predominant gut microbial formation in $\mathrm{CN}+$ was almost consistent with that in $\mathrm{CN}-$.

LEfSe analysis was used to identify the distinct microbiota of $\mathrm{CN}+$ participants. The results showed that the relative abundance of phylum Bacteroidetes, class Bacteroidia, and order Bacteroidales were significantly enriched, whereas phylum Firmicutes, class Clostridia, class Deltaproteobacteria, order Clostridiales, order Desulfovibrionales, family Lachnospiraceae, family Desulfovibrionaceae, family Ruminococcaceae, genus Bilophila, and genus Faecalibacterium were significantly reduced in the $\mathrm{CN}+$ group (LDA score cutoff $>2.0$, Fig. $3 \mathrm{~B}$ ). The relative abundance and distribution of these selected microbiota were presented in a heatmap (Fig. 3C).

In addition, the GLMs were also used to evaluate the differences of these gut microbiota identified by the LEfSe. We found that no significant differences were 


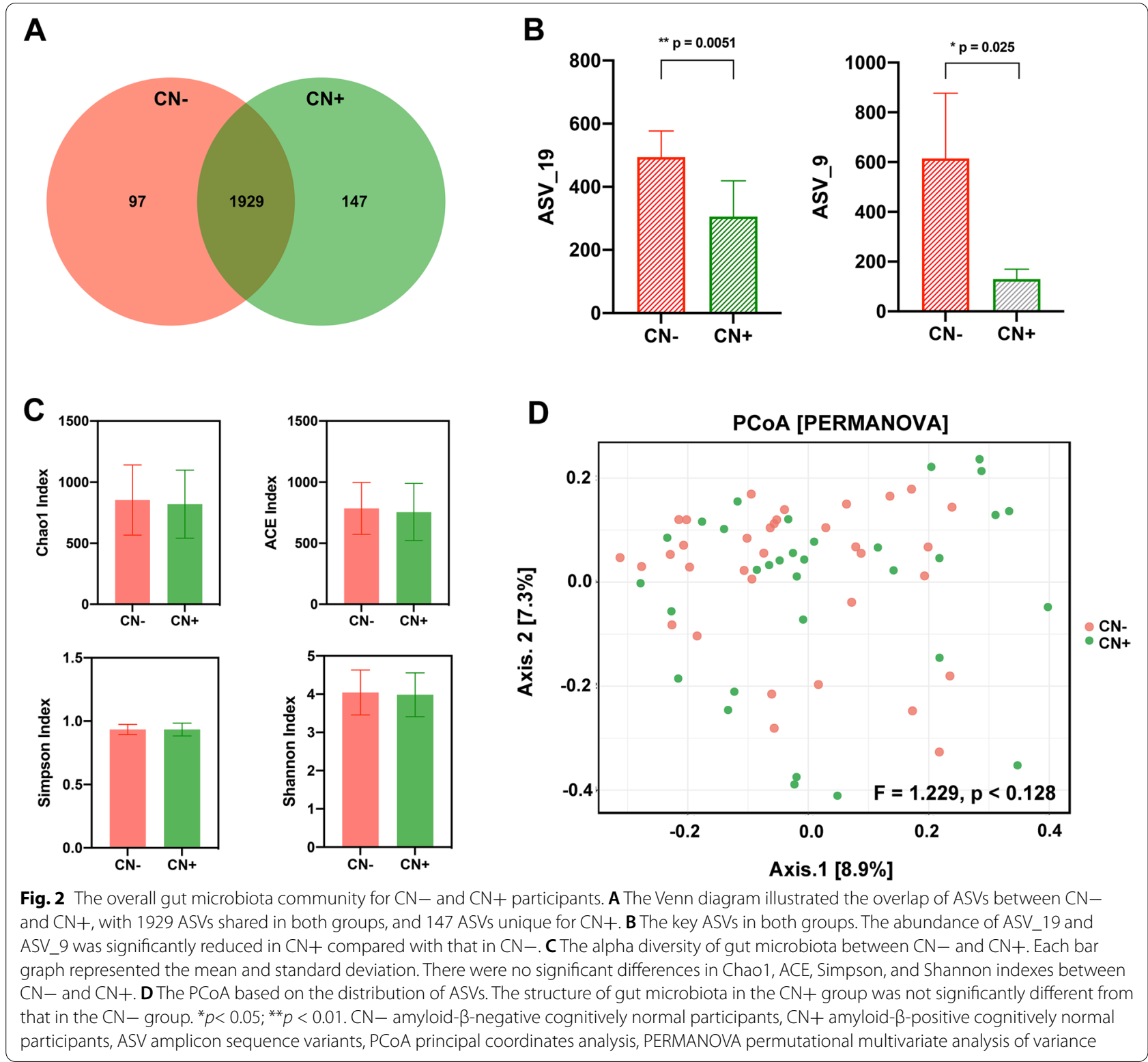

found in family Ruminococcaceae $(p=0.067)$ and genus Bilophila $(p=0.177)$ after controlling for age, sex, BMI, and APOE (Table 2). For taxa with the relative abundance $\geq 1 \%$, the relative abundance of family Ruminococcaceae and genus Faecalibacterium were not significant after correction for multiple comparisons $(p>0.05)$, whereas abundant differences of order Bacteroidales and family Lachnospiraceae were marginal in statistical significance ( $p=0.070$ and $p=0.066$, respectively) (Supplementary Table S3). Meanwhile, no significant interaction effect was found with APOE genotype and the $A \beta$ status in the altered gut microbiota for $\mathrm{CN}-$ and $\mathrm{CN}+$ participants (Supplementary Table S4).
Furthermore, the relative abundance of altered gut microbiota among the $\mathrm{CN}-, \mathrm{CN}+$, and $\mathrm{CI}$ groups are shown in Figure S3. We found that the phylum Firmicutes and its corresponding class Clostridia, order Clostridiales, family Lachnospiraceae, family Ruminococcaceae, genus Lachnospiracea_incertae_sedis, and genus Faecalibacterium taxa showed a progressive decline from $\mathrm{CN}-$ to $\mathrm{CN}+$ and $\mathrm{CI}$.

\section{The association of plasma $A \beta$ markers and gut microbiota with brain $A \beta$ burden}

In our study, we found that the global brain SUVR was negatively associated with plasma $A \beta_{42} / A \beta_{40}$ for all $C N$ participants $(r=$ 

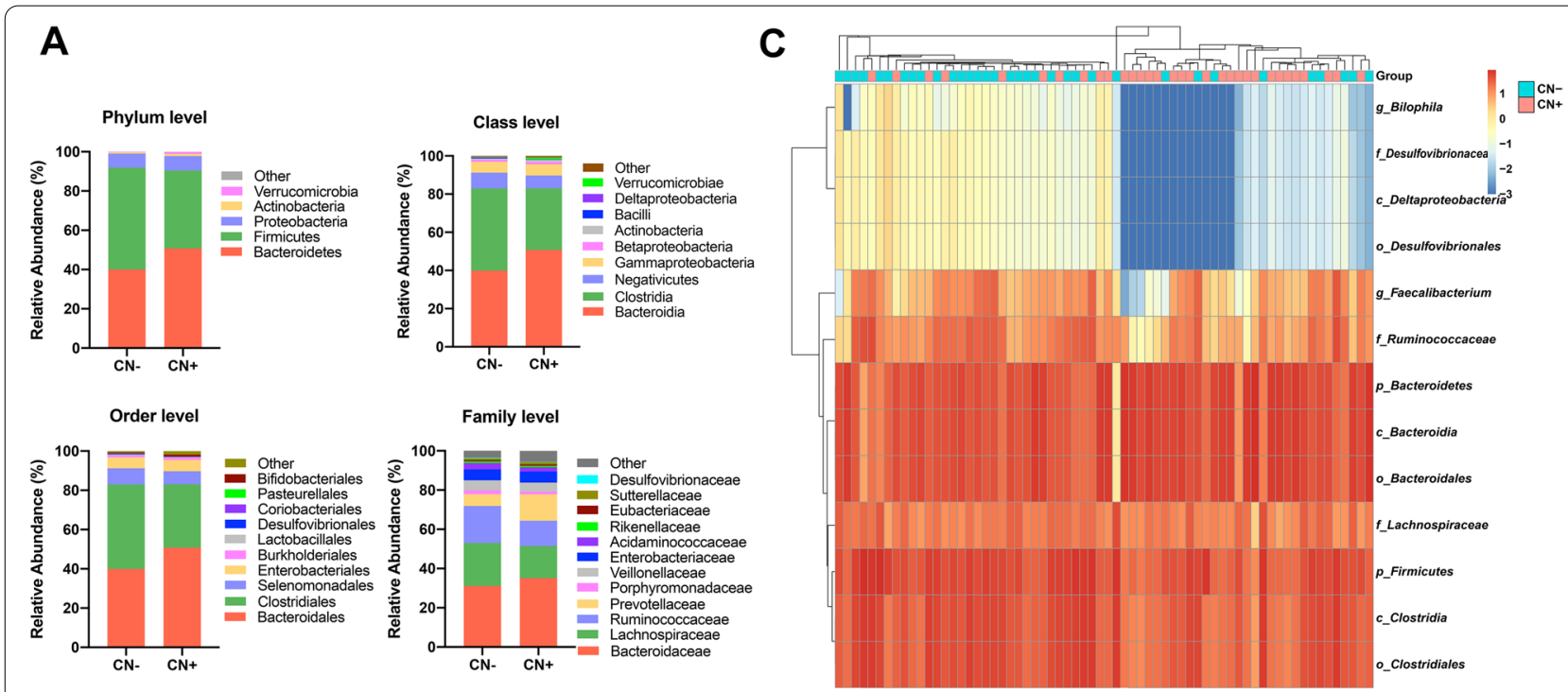

B

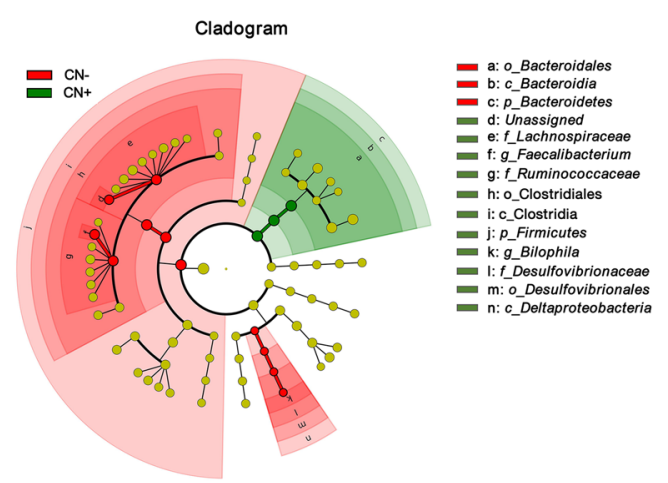

Fig. 3 The gut microbial compositions for $\mathrm{CN}$ - and $\mathrm{CN}+$ participants. A The bacterial community in both groups at different taxonomic levels. Bar graphs indicated the relative abundance of phylum-level, class-level, order-level, and family-level taxa. $\mathbf{B}$ LEfSe analysis between CN- and $\mathrm{CN}+$. As shown in the histogram of LDA scores for differentially abundant taxa, positive LDA scores indicated the enrichment of taxa in the $\mathrm{CN}+$ group (green), and negative LDA scores indicated the enriched taxa in the CN-group (red). The LDA scores $(\log 10)>2$ and $p<0.05$ were listed. Cladogram indicated the phylogenetic distribution of gut bacteria. Colors represented different groups (CN-, red; CN+, green). Nodes with different colors represented important taxa in different groups. Yellow nodes suggested no significantly differential taxa between the two groups. C The heatmap showing the relative abundance and distribution of differentially abundant taxa identified by the LEfSe method. CNamyloid- $\beta$-negative cognitively normal participants, $C N+$ amyloid- $\beta$-positive cognitively normal participants, LEfSe linear discriminant analysis (LDA) effect size

$-0.298, p=0.015)$ (Fig. 4A). However, no significant association with plasma $\mathrm{A} \beta_{42}$ and $\mathrm{A} \beta_{40}$ was observed $(r=-0.209, p=0.093$; $r=-0.085, p=0.499$, respectively). Subsequently, we investigated the association of altered gut microbiota with the brain $A \beta$ burden. As shown in Fig. 4B-D, the log10-transformed family Desulfovibrionaceae, genus Bilophila, and genus Faecalibacterium was negatively correlated with the global brain $\operatorname{SUVR}(r=-0.331, p=$ $0.007 ; r=-0.247, p=0.046 ; r=-0.291, p=0.018$, respectively).

\section{The discriminative power of the combined gut microbiota and plasma $A \beta$}

Using the ROC analysis approach, we first estimated the discriminative power of each of the plasma $A \beta$ markers in identifying individuals with $\mathrm{CN}+$ from $\mathrm{CN}-$. The plasma $A \beta_{42} / A \beta_{40}$ showed a relatively good discriminative power, followed by the plasma $\mathrm{A} \beta_{42}$, with AUCs of 0.715 (95\%: $0.591 \sim 0.838$ ) and 0.660 (95\%: $0.528 \sim 0.792$ ), respectively. At the cutoff optimized for balanced sensitivity and specificity (Youden's Index), the sensitivity of all single plasma markers was between $71.88 \%$ and $81.25 \%$, whereas the specificity was $52.94 \%$ and $67.65 \%$ for plasma $A \beta_{42}$ and $A \beta_{42} / A \beta_{40}$, respectively, and only $32.25 \%$ for plasma $A \beta_{40}$. Then, the combination of the plasma $A \beta_{40}, A \beta_{42}$, and $A \beta_{42} / A \beta_{40}$ further improved the discriminative power (panel 1: AUC $=0.730,95 \%$ : 0.608 $\sim 0.852$; Table 3; Fig. 5A). 
Table 2 Alterations of the gut microbiota between $\mathrm{CN}-$ and $\mathrm{CN}+$ using GLM

\begin{tabular}{lllll}
\hline & $\mathbf{C N}-(\boldsymbol{n}=\mathbf{3 4})$ & $\mathbf{C N}+(\boldsymbol{n}=\mathbf{3 2})$ & $\boldsymbol{F}_{(\mathbf{1}, \mathbf{6 0})}$ & $\boldsymbol{P}$ value \\
\hline P_Bacteroidetes & $40.01 \pm 16.14$ & $50.76 \pm 18.25$ & 7.103 & 0.010 \\
P_Firmicutes & $51.85 \pm 16.08$ & $39.71 \pm 17.26$ & 7.768 & 0.007 \\
C_Bacteroidia & $40.00 \pm 16.15$ & $50.76 \pm 18.25$ & 7.109 & 0.010 \\
C_Clostridia & $42.93 \pm 15.01$ & $32.26 \pm 16.71$ & 5.992 & 0.017 \\
C_Deltaproteobacteria & $0.39 \pm 0.51$ & $0.15 \pm 0.25$ & 4.472 & 0.039 \\
O_Bacteroidales & $40.00 \pm 16.15$ & $50.76 \pm 18.25$ & 7.109 & 0.010 \\
O_Clostridiales & $42.93 \pm 15.01$ & $32.26 \pm 16.71$ & 5.992 & 0.017 \\
O_Desulfovibrionales & $0.39 \pm 0.51$ & $0.15 \pm 0.25$ & 4.472 & 0.039 \\
f_Lachnospiraceae & $21.92 \pm 9.50$ & $16.33 \pm 7.19$ & 6.096 & 0.016 \\
f_Ruminococcaceae & $18.86 \pm 11.79$ & $12.93 \pm 11.82$ & 3.491 & 0.067 \\
f_Desulfovibrionaceae & $0.39 \pm 0.51$ & $0.15 \pm 0.25$ & 4.472 & 0.039 \\
g_Bilophila & $0.29 \pm 0.50$ & $0.13 \pm 0.24$ & 1.863 & 0.177 \\
g_Faecalibacterium & $11.41 \pm 8.65$ & $7.08 \pm 7.99$ & 4.008 & 0.050 \\
\hline
\end{tabular}

Statistical analysis was conducted using GLM, with age, sex, BMI, and APOE as possible confounding factors

Abbreviations: GLM general linear model, $C N$ - amyloid- $\beta$-negative cognitively normal participants, $C N+$ amyloid- $\beta$-positive cognitively normal participants, $\mathrm{Cl}$ cognitive impairment, $p$ phylum, $c$ class, $o$ order, $f$ family, $g$ genus

We also calculated the classification power of each of the gut taxa, as well as that of the combined gut taxa for distinguishing $\mathrm{CN}+$ from $\mathrm{CN}-$. We defined the combined phylum Bacteroidetes, class Bacteroidia, and order Bacteroidales as the taxa 1; the combined phylum Firmicutes, class Clostridia, order Clostridiales, family Lachnospiraceae, family Ruminococcaceae, and genus Faecalibacterium as the taxa 2; the combined class Deltaproteobacteria, order Desulfovibrionales, family Desulfovibrionaceae, and genus Bilophila as the taxa 3 . Compared with the single taxa, the combination of the taxa1, taxa2, and taxa3 (panel 2) showed relatively higher classification accuracy, with an AUC of 0.810 (95\%: 0.707 $\sim 0.912$; Table 3; Fig. 5B). In addition, the discriminative power of the combined cognitive tests (including MoCAB, AVLT-long delayed recall, AVLT-R, STT-A, STT-B, AFT, BNT, panel 3) was relatively good, with an AUC of 0.802 (95\%: $0.691 \sim 0.912$; Table 3). Finally, as is shown in Fig. 5C and Table 3, compared with the panel 1, the AUC of the panel 4 improved to 0.869 (95\% CI 0.782 $0.955)$ after adding the panel 2 and panel $3(p=0.0086$, DeLong' test), suggesting that the combination of plasma markers, gut microbiota, and cognition reached an optimal classification.

\section{Discussion}

In this study, we characterized the gut microbiota in the preclinical stage of $\mathrm{AD}$ and further investigated the potential classification efficiency of the combined gut microbiota and plasma $A \beta$ markers for identifying $C N$ individuals with brain amyloidosis. We found that plasma $A \beta_{42}$ and $A \beta_{42} / A \beta_{40}$ significantly reduced in the $C N+$ group relative to the $\mathrm{CN}-$ group. The relative abundance of phylum Bacteroidetes were enriched, whereas taxa in Firmicutes and Proteobacteria phyla were reduced in $\mathrm{CN}+$. In addition, the global brain, $\mathrm{A} \beta$ burden was negatively associated with the plasma $A \beta_{42} / A \beta_{40}$, family Desulfovibrionaceae, genus Bilophila and genus Faecalibacterium for all $\mathrm{CN}$ participants. Importantly, the combination of plasma $A \beta$ markers, altered gut microbiota, and clinical cognition showed the potential of distinguishing $\mathrm{CN}+$ from $\mathrm{CN}-$, suggesting that the combined gut microbiota and plasma $A \beta$ markers may serve as a minimally invasive and cost-effective index for screening preclinical AD.

In the present study, we found that the level of plasma $\mathrm{A} \beta_{42}$ and $A \beta_{42} / A \beta_{40}$ in $\mathrm{CN}+$ was lower than that in $\mathrm{CN}-$. Besides, plasma $A \beta_{42} / A \beta_{40}$ was negatively associated with brain $A \beta$ burden, whereas plasma $A \beta_{42}$ and $A \beta_{40}$ showed no correlation with brain amyloidosis. Our results indicated that the ratio of $A \beta_{42}$ and $A \beta_{40}$ appeared to be more predictive of brain $A \beta$ pathological changes than $A \beta_{42}$ and $A \beta_{40}$. Currently, the published results regarding the correlation between plasma $A \beta$ and $A D$ are conflicting. Many studies evaluated plasma $A \beta_{42}$ as a biomarker of $\mathrm{AD}[15,16,35,36]$, whereas a systematic review and meta-analysis comprising 231 articles reported that plasma $A \beta_{42}$ and $A \beta_{40}$ were not associated with $A D$ [37]. However, recent reports have demonstrated that plasma $\mathrm{A} \beta_{42} / \mathrm{A} \beta_{40}$ are strongly predictive of brain amyloidosis, even in individuals with $\mathrm{CN}+[20,36]$, which was consistent with our findings. Schindler also pointed out that plasma $A \beta_{42} / A \beta_{40}$ had higher concordance with brain amyloidosis than plasma $A \beta_{42}$ and $A \beta_{40}$ separately. One possible explanation is that $A \beta_{42} / A \beta_{40}$ may normalize for preanalytical variability or differences in $A \beta$ levels related to circadian rhythms or other biological variation not related to brain amyloidosis. In the current study, plasma $A \beta$ markers, especially $A \beta_{42} / A \beta_{40}$, had the potential to distinguish $\mathrm{CN}+$ individuals from $\mathrm{CN}-(\mathrm{AUC}=$ $71.5 \%$ ). This accuracy is highly comparable to what was reported in another study that the diagnostic accuracy of $A \beta_{42} / A \beta_{40}$ measurement alone for amyloid PET positivity was reasonably good with an AUC of 73.0\% [38]. Since the changed plasma $A \beta$ levels are rather small, we thought that the combination of multiple markers may assist in achieving a better discriminative power between $\mathrm{CN}+$ and $\mathrm{CN}-$. Therefore, a model including $\mathrm{A} \beta_{42} / \mathrm{A} \beta_{40}$, $A \beta_{42}$ and $A \beta_{40}$ improved the diagnostic efficiency, with an AUC of $73.0 \%$.

In our study, individuals with $\mathrm{CN}+$ showed similar gut microbial alterations like $\mathrm{AD}$, suggesting that changes of the gut microbial profiling occurred in the 

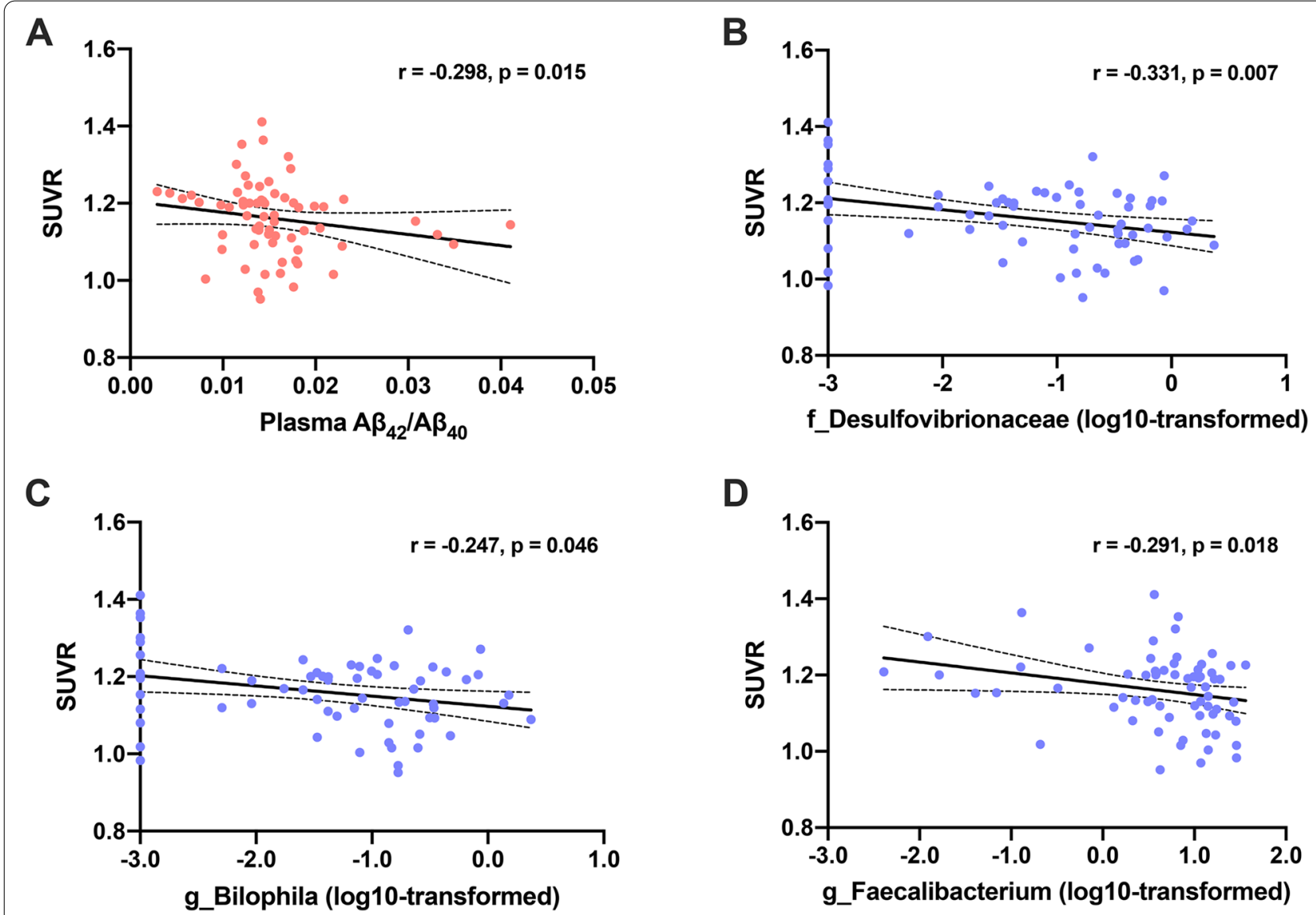

Fig. 4 The association of plasma $A \beta$ markers and gut microbiota with global brain $A \beta$ burden. $\mathbf{A}$ There was negative correlation between global SUVR and plasma $A \beta_{42} / A \beta_{40}$. The family Desulfovibrionaceae (B), genus Bilophila (C), and genus Faecalibacterium (D) were negatively correlated with the global SUVR. $A \beta$ amyloid- $\beta$, SUVR standard uptake value rate

preclinical AD. Previous studies have demonstrated markedly decreased phylum Firmicutes in AD patients compared with healthy controls, which was similar to our findings. To the best of our knowledge, phylum Firmicutes is responsible for the regulation of inflammatory responses and human metabolic functions, which may in turn affect behavior and cognition $[39,40]$. Accumulating evidence has indicated that intestinal dysbiosis has an adverse impact on the human neuroinflammation, which further contributes to the occurrence and progression of AD [41-43]. The depletion of phylum Firmicutes may promote the production of pro-inflammatory cytokines and toxic metabolites, and meanwhile, reduce the quantity of beneficial substances such as short-chain fatty acids (SCFAs), leading to the damage of gut epithelial barrier and subsequent blood-brain barrier (BBB) dysfunctions [44]. In addition, the relative abundance of class Clostridia, order Clostridiales, family Ruminococcaceae and family Lachnospiraceae, which is key SCFA-producing bacteria belonging to phylum
Firmicutes, was also significantly reduced in $\mathrm{CN}+$ participants. Current studies based on animal models have suggested the inner correlation between the decreased gastrointestinal SCFA level and the onset of AD [45]. SCFAs are the gut microbial-derived metabolites, which are mainly from dietary components that are incompletely hydrolyzed due to a lack of appropriate enzymes $[6,46]$. SCFAs may be strongly involved in glucose regulation in humans and have beneficial effects on energy homeostasis and metabolism [47, 48]. Researchers have proposed that SCFAs play a critical role in the maintenance of homeostasis within the central nervous system [49] and ameliorating the BBB permeability [50]. The family Ruminococcaceae and family Lachnospiraceae are also related to insulin resistance, which is regarded as a high-risk factor for developing $\mathrm{AD}$ [51]. In this study, the results also revealed that genus Faecalibacterium was negatively correlated with brain $\mathrm{A} \beta$ deposition. To our knowledge, the genus Faecalibacterium and its metabolites butyrate have anti-inflammatory effects. 
Table 3 The AUC and sensitivity and specificity at Youden's cutoff to identify CN+ participants

\begin{tabular}{|c|c|c|c|c|c|}
\hline & AUC (95\% Cl) & Youden's cut point & Sensitivity (\%) & Specificity (\%) & $P$ value \\
\hline \multicolumn{6}{|l|}{ Plasma $A \beta$ markers } \\
\hline Plasma $A \beta_{40}$ & $0.539(0.399 \sim 0.679)$ & $663.7 \mathrm{pg} / \mathrm{mL}$ & 81.25 & 32.35 & 0.586 \\
\hline Plasma $A \beta_{42}$ & $0.660(0.528 \sim 0.792)$ & $11.69 \mathrm{pg} / \mathrm{mL}$ & 81.25 & 52.94 & 0.025 \\
\hline Plasma $A \beta_{42} / A \beta_{40}$ & $0.715(0.591 \sim 0.838)$ & 0.015 & 71.88 & 67.65 & 0.003 \\
\hline Panel 1 & $0.730(0.608 \sim 0.852)$ & 0.510 & 68.75 & 73.53 & 0.001 \\
\hline \multicolumn{6}{|l|}{ Gut microbiota } \\
\hline Taxa1 & $0.686(0.555 \sim 0.817)$ & 0.500 & 68.75 & 67.65 & 0.009 \\
\hline Taxa2 & $0.775(0.663 \sim 0.887)$ & 0.625 & 56.25 & 88.24 & $<0.001$ \\
\hline Taxa3 & $0.734(0.613 \sim 0.855)$ & 0.574 & 68.75 & 73.53 & 0.001 \\
\hline Panel 2 & $0.810(0.707 \sim 0.912)$ & 0.383 & 87.50 & 64.71 & $<0.001$ \\
\hline \multicolumn{6}{|l|}{ Cognitive scores } \\
\hline Panel 3 & $0.802(0.691 \sim 0.912)$ & 0.573 & 68.75 & 88.24 & $<0.001$ \\
\hline \multicolumn{6}{|l|}{ Combined model } \\
\hline Panel 4 & $0.869(0.782 \sim 0.955)$ & 0.356 & 87.50 & 73.53 & $<0.001$ \\
\hline
\end{tabular}

Taxa 1, combined phylum Bacteroidetes, class Bacteroidia, and order Bacteroidales

Taxa 2, combined phylum Firmicutes, class Clostridia, order Clostridiales, family Lachnospiraceae, family Ruminococcaceae, and genus Faecalibacterium

Taxa 3, combined class Deltaproteobacteria, order Desulfovibrionales, family Desulfovibrionaceae, and genus Bilophila

Panel 1, the combined plasma $A \beta_{40}, A \beta_{42}$, and $A \beta_{42} / A \beta_{40}$

Panel 2, the combined taxa 1, taxa 2, and taxa 3

Panel 3, the combined clinical cognitive tests (MoCA-B, AVLT-long delayed recall, AVLT-R, STT-A, STT-B, AFT, BNT)

Panel 4, the combined plasma $A \beta$ markers, gut taxa, and cognitive tests

Abbreviations: $A \cup C$ area under curve, $A \beta$ amyloid- $\beta, C N+$ amyloid- $\beta$-positive cognitively normal participants

The reduced genus Faecalibacterium may lead to the decreased anti-inflammatory role, which further results in $\mathrm{AD}$ pathology. It is noteworthy that there was a progressively declined trend in phylum Firmicutes, as well as its corresponding class, order, family, and genus taxa from $\mathrm{CN}-$ to $\mathrm{CN}+$ and $\mathrm{CI}$, indicating again that the gut microbial alterations in preclinical $\mathrm{AD}$ might be at an intermediate stage in the $\mathrm{AD}$ continuum.
We also found the significantly enriched phylum $\mathrm{Bac}$ teroidetes and its relatives in $\mathrm{CN}+$ participants, but no progressively increased Bacteroidetes in CI patients when compared to $\mathrm{CN}-$ individuals. The previously published literatures regarding the association of Bacteroidetes with AD are contradictory. For instance, Nicholas M. Vogt et al. reported significantly increased bacterial abundance in Bacteroidetes [12], whereas Zhuang and his colleagues
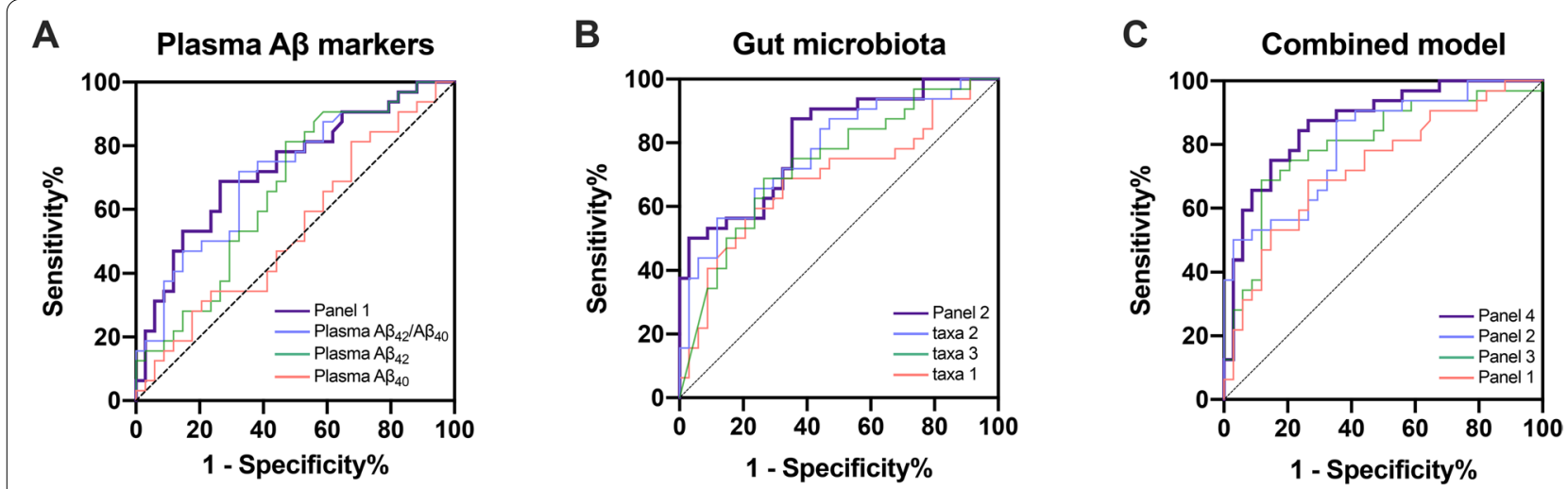

Fig. 5 ROCs for $\mathrm{CN}+$ participants. A The discriminative power of individual plasma $\mathrm{A} \beta$ markers and the combined panel in identifying $\mathrm{CN}+$ from $\mathrm{CN}-$; $\mathbf{B}$ The discriminative power of each of the gut taxa and the combined panel in identifying $\mathrm{CN}+$ from $\mathrm{CN}-; \mathbf{C}$ The predicted values of the combined panels in identifying $C N+$ from $C N-$. $A \beta$, amyloid- $\beta ; C N-$, amyloid- $\beta$-negative cognitively normal participants; $C N+$, amyloid- $\beta$-positive cognitively normal participants; panel 1, the combined plasma $A \beta_{40}, A \beta_{42}$, and $A \beta_{42} / A \beta_{40}$; panel 2, the combined taxa 1, taxa 2, and taxa 3; panel 3, the combined clinical cognitive tests (including MoCA-B, AVLT-long delayed recall, AVLT-R, STT-A, STT-B, AFT, BNT); panel 4, the combined plasma A $\beta$ markers, gut taxa, and cognitive tests 
found a mild decrease in the abundance of Bacteroidetes among $\mathrm{AD}$ patients [52]. Interestingly, one study characterizing the gut microbiota in the prodromal stage of AD found that Bacteroidetes was significantly enriched in amnestic MCI patients and unexpectedly decreased in $\mathrm{AD}$ patients to the normal level [11]. Likewise, in our study, individuals in the preclinical stage of AD showed the highest abundance in phylum Bacteroidetes among the $\mathrm{CN}-, \mathrm{CN}+$, and $\mathrm{CI}$ groups. The phylum Bacteroidetes encompasses a diverse group of gram-negative commensal bacteria in the gut [53], whose major outer membrane component is lipopolysaccharide (LPS). LPS plays a crucial role in triggering systemic inflammation and the release of pro-inflammatory cytokines, which can further lead to brain amyloid deposition [54, 55]. Thus, the enriched phylum Bacteroidetes and its relatives in $\mathrm{CN}+$ may result in increased translocation of LPS from the gut to systemic circulation, which in turn may exacerbate AD pathology through inflammation or other mechanisms [12].

Moreover, class Deltaproteobacteria, as well as its corresponding order, family, and genus taxa were significantly decreased in $\mathrm{CN}+$ individuals. Class Deltaproteobacteria is the fourth described class of the phylum Proteobacteria, including a series of sulfate-reducing bacteria $[53,56]$. The metabolic end-product of these bacteria is the hydrogen sulfide, the overproduction of which in the gastrointestinal tract has been linked to ulcerative colitis and colon cancer [57]. Additionally, in our study, we also found negative associations of class Deltaproteobacteria, order Desulfovibrionales, family Desulfovibrionaceae, genus Bilophila with brain A $\beta$ burden, possibly providing a clue that Deltaproteobacteria may contribute to AD pathology. However, the correlation between Deltaproteobacteria and AD is largely unclear. A prior study reported the enriched proteobacteria in $\mathrm{AD}$ and MCI patients compared with healthy controls, which focused on class Gammaproteobacteria and family Enterobacteriaceae [11]. The discrepancy of different studies may be attributed to differences in sample size, population, RNA sequencing method, and comorbidity condition.

In this study, we also assessed the discriminative power of these altered gut microbiota. The taxa 2 showed a relatively good discriminative power, followed by the taxa 3 and taxa 1, with AUCs of 0.775, 0.734, and 0.686, respectively. Furthermore, the combination of three taxa showed a relatively higher classification accuracy, with an AUC of 0.810. When we underwent the simultaneous evaluation of plasma $A \beta$ markers, gut microbiota, and clinical cognitive scores, the discriminative power reached a larger diagnostic accuracy for $\mathrm{CN}+$ individuals $($ AUC $=0.869)$, suggesting that combination of plasma
$\mathrm{A} \beta$ and gut microbiota provides a potentially valuable tool for the identification of amyloid PET status.

Our study showed a very slight non-significant trend of decreased alpha diversity in $\mathrm{CN}+$ compared with that in $\mathrm{CN}-$. After adding the $\mathrm{CI}$ group, the alpha diversity expressed as Chaol and ACE was lower in CI than in $\mathrm{CN}-$, which was in agreement with the previous studies [12]. Nevertheless, in one study by Liu et al., although the whole alpha diversity also decreased in $\mathrm{AD}$, indexes with statistical significance mainly in Shannon and Simpson [11]. Moreover, the Chao1, ACE, and Shannon indexes of $\mathrm{CN}+$ individuals were at an intermediate stage between $\mathrm{CN}-$ and $\mathrm{CI}$ in our study. Notably, the pathophysiological mechanism of altered alpha diversity still needs further investigation. For the beta diversity, the gut taxonomic composition of CI was significantly different from that of $\mathrm{CN}-$ using the NMDS, while there were no gut taxonomic differences between $\mathrm{CN}-$ and $\mathrm{CN}+$, and between $\mathrm{CN}+$ and $\mathrm{CI}$. Our findings were consistent with the previous reports that the fecal microbial structure in AD was significantly different from that of controls $[11$, $12,52]$.

\section{Limitations}

Here, there are also some limitations which warrant attention. Firstly, this is a preliminary, single-center study and the sample size is relatively small. In future studies, a larger sample size from multiple centers is essential to provide more evidences. Secondly, although the participants in our study have been matched in the demography, nationality, place of residence and lifestyles, the effect of other potential factors (e.g., medications, external stressors, immune function) is still difficult to control. Cryan et al. consider that most of the current gut-related studies are underpowered, with participant-selection bias, inconsistent sample size, different sequencing protocols, bioinformatics pipelines, and statistical methods [5]. Thus, to objectively mirror the intrinsic relation of the microbiota-gut-brain axis, more standardized and well-designed studies are needed in the future. Thirdly, in our study, not all the CI patients had amyloid-PET data, and the diagnosis of $\mathrm{MCI}$ and $\mathrm{AD}$ was mainly based on the clinical practice $[58,59]$. Therefore, in the future, CI patients with evidence of brain amyloidosis are necessary to be recruited to provide more accurate evidence of the gut microbiota in the spectrum of $\mathrm{AD}$. Finally, 16S rDNA amplicon sequencing analysis can only reach genus-level resolution, and it is more sensitive to the specific primers and number of PCR cycles chosen. Metagenomic sequencing analysis, characterized by extending taxonomic resolution to the species- or strainlevel and simultaneously providing potential functional 
information, will provide more microbial information [60]. In the future work, the combination of the $16 \mathrm{~S}$ rDNA amplicon sequencing and metagenomic sequencing techniques can be used.

\section{Conclusions}

In summary, this study characterized the gut microbiota in individuals with preclinical $A D$ and further illuminated the association of gut microbiota and plasma $A \beta$ with brain amyloidosis. Our findings supported that the combination of gut microbiota and plasma $A \beta_{42} /$ $A \beta_{40}$ may be used as a screening tool for preclinical AD and targeting gut microbiota may offer novel thoughts towards the therapeutic strategies of AD-related cognitive decline.

\begin{abstract}
Abbreviations
AD: Alzheimer's disease; Aß: Amyloid- $\beta$; AFT: Animal Fluency Test; AGE:

Agarose gel electrophoresis; APOE: Apolipoprotein E; ASV: Amplicon sequence variants; AVLT-H: Auditory Verbal Learning Test-HuaShan version; BMI: Body mass index; BNT: Boston Naming Test; CN: Cognitively normal; CSF: Cerebrospinal fluid; FAQ: Functional Activities Questionnaire; HAMA: Hamilton Anxiety Rating Scale; HAMD: Hamilton Depression Rating Scale; LEfSe: Linear discriminant analysis LDA effect size; LPS: Lipopolysaccharide; MCI: Mild cognitive impairment; NIA-AA: National Institute on Aging and Alzheimer's Association; MoCA-B: Montreal Cognitive Assessment-Basic Version; MSD: Meso Scale Discovery; PCoA: Principal coordinate analysis; PCR: Polymerase chain reaction; PERMANOVA: Permutational multivariate analysis of variance; PET: Positron emission tomography; RDP: Ribosomal Database Project; SCD: Subjective cognitive decline; SCFAs: Short-chain fatty acids; SILCODE: Sino Longitudinal Study on Cognitive Decline; STT-A: Shape Trails Test Part A; STT-B: Shape Trails Test Part B; SUVR: Standard uptake value rate.
\end{abstract}

\section{Supplementary Information}

The online version contains supplementary material available at https://doi. org/10.1186/s13195-022-00977-x.

Additional file 1 : Supplementary Table $\mathbf{S 1}$. The items of the semi-structured interview for lifestyles. Supplementary Table S2. Demographic information and neuropsychological assessments for $\mathrm{Cl}$ patients. Supplementary Table S3. Gut microbial differences between $\mathrm{CN}$ - and $\mathrm{CN}+$ with correction for multiple comparisons. Supplementary Table S4. The interaction effect between APOE and diagnosis in the altered gut microbiota for $\mathrm{CN}$ - and $\mathrm{CN}+$ participants. Supplementary Figure S1. The alpha diversity of gut microbiota among the $\mathrm{CN}-, \mathrm{CN}+$, and $\mathrm{Cl}$ groups. Each bar graph represented the mean and standard deviation. The Chao 1 and ACE indexes showed significantly decline in $\mathrm{Cl}$ compared with $\mathrm{CN}$ - There were no significant differences in Simpson and Shannon indexes among the three groups. ${ }^{*}, p<0.05$. $C N$-, amyloid- $\beta$ negative cognitively normal participants; $\mathrm{CN}+$, amyloid- $\beta$ positive cognitively normal participants; $\mathrm{Cl}$, cognitive impairment participants. Supplementary Figure S2. The PCoA and NMDS based on the distribution of ASVs. (A) PCoA showed that the difference of gut taxonomic composition between $\mathrm{CN}$ - and $\mathrm{Cl}$ was marginal in statistical significance ( $F=1.383, p<0.052)$; (B) NMDS showed that the gut taxonomic composition was significantly different between $\mathrm{CN}$ - and $\mathrm{Cl}(\mathrm{R}=0.097, p<0.009)$; (C) PCoA showed that the gut taxonomic composition between $\mathrm{CN}+$ and $\mathrm{Cl}$ was not significantly different $(\mathrm{F}=0.850$, $p<0.712$ ); (D) NMDS showed that the structure of gut microbiota in the $\mathrm{CN}+$ group was not significantly different from that in the $\mathrm{Cl}$ group ( $\mathrm{F}=$ $-0.020, p<0.698)$. $C N-$, amyloid- $\beta$ negative cognitively normal participants; $\mathrm{CN}+$, amyloid- $\beta$ positive cognitively normal participants; $\mathrm{Cl}$, cognitive impairment participants; ASV, amplicon sequence variants; PCoA, principal coordinates analysis; PERMANOVA, permutational multivariate analysis of variance; NMDS, non-metric multidimensional scaling; ANOSIM, analysis of similarities. Supplementary Figure S3. The relative abundance of altered gut microbiota at different taxonomic levels among the $\mathrm{CN}-, \mathrm{CN}+$ and $\mathrm{Cl}$ groups. Bar graphs indicated the relative abundance of phylum-level (A), class-level (B), order-level (C), family-level (D), and genus-level (E) taxa. The phylum Firmicutes and its corresponding class Clostridia, order Clostridiales, family Desulfovibrionaceae, family Ruminococcaceae, genus Lachnospiracea_incertae_sedis and genus Faecalibacterium taxa showed a progressive decline from $\mathrm{CN}$ - to $\mathrm{CN}+$ and $\mathrm{Cl}$. $\mathrm{CN}$-, amyloid- $\beta$ negative cognitively normal participants; $\mathrm{CN}+$, amyloid- $\beta$ positive cognitively normal participants; $\mathrm{Cl}$, cognitive impairment participants.

\section{Acknowledgements}

We thank all participants who donated their samples.

\section{Authors' contributions}

Y. Han and C. Sheng designed the study; W. Du and K. Yang performed the statistical analysis; C. Sheng and B. He drafted the initial manuscript; Y. Cai was responsible for the blood sample collection and analysis. The authors contributed to revision and editing of the final manuscript.

Funding

This article was supported by the National Natural Science Foundation of China (Grant No. 61633018, 82020108013, 81801052).

\section{Availability of data and materials}

All microbiome sequence data are available upon request from the authors.

\section{Declarations}

Ethics approval and consent to participate

This study was registered on ClinicalTrials.gov (Identifier: NCT03370744), and research activities were approved by the Medical Research Ethics Committee and Institutional Review Board of Xuanwu Hospital in the Capital Medical University (ID: [2017]046). Each participant needed to provide a written informed consent before participating in study procedures.

\section{Consent for publication}

Not applicable.

\section{Competing interests}

The authors declare that they have no competing interests.

\section{Author details}

${ }^{1}$ Department of Neurology, Xuanwu Hospital of Capital Medical University, Beijing 100053, China. ${ }^{2}$ Evidence-Based Medicine Center, Xuanwu Hospital of Capital Medical University, Beijing 100053, China. ${ }^{3}$ Department of Epidemiology and Biostatistics, School of Public Health, Capital Medical University, Beijing 100069, China. ${ }^{4}$ Key Laboratory of Biomedical Engineering of Hainan Province, School of Biomedical Engineering, Hainan University, Haikou 570228, China. ${ }^{5}$ Department of Neurobiology, Xuanwu Hospital of Capital Medical University, Beijing 100053, China. ${ }^{6}$ Key Laboratory for Neurodegenerative Diseases of the Ministry of Education, Beijing 100053, China. ${ }^{7}$ Department of Biobank, Xuanwu Hospital of Capital Medical University, Beijing 100053, China. ${ }^{8}$ Center of Alzheimer's Disease, Beijing Institute for Brain Disorders, Beijing 100053 , China. ${ }^{9}$ National Clinical Research Center for Geriatric Diseases, Beijing 100053, China.

Received: 6 September 2021 Accepted: 6 February 2022

Published online: 14 February 2022

\section{References}

1. Jia J, Wei C, Chen S, Li F, Tang Y, Qin W, et al. The cost of Alzheimer's disease in China and re-estimation of costs worldwide. Alzheimers Dement. 2018;14:483-91. 
2. Jack CR Jr, Bennett DA, Blennow K, Carrillo MC, Dunn B, Haeberlein SB, et al. NIA-AA research framework: toward a biological definition of Alzheimer's disease. Alzheimers Dement. 2018;14:535-62.

3. Wang $X$, Huang W, Su L, Xing Y, Jessen F, Sun Y, et al. Neuroimaging advances regarding subjective cognitive decline in preclinical Alzheimer's disease. Mol Neurodegener. 2020;15:55.

4. Scheltens P, Blennow K, Breteler MM, de Strooper B, Frisoni GB, Salloway S, et al. Alzheimer's disease. Lancet. 2016;388:505-17.

5. Cryan JF, O'Riordan KJ, Sandhu K, Peterson V, Dinan TG. The gut microbiome in neurological disorders. Lancet Neurol. 2020;19:179-94.

6. Cryan JF, O'Riordan KJ, Cowan CSM, Sandhu KV, Bastiaanssen TFS, Boehme M, et al. The microbiota-gut-brain axis. Physiol Rev. 2019;99:1877-2013.

7. Sherwin E, Dinan TG, Cryan JF. Recent developments in understanding the role of the gut microbiota in brain health and disease. Ann NY Acad Sci. 2018;1420:5-25.

8. Cattaneo A, Cattane N, Galluzzi S, Provasi S, Lopizzo N, Festari C, et al. Association of brain amyloidosis with pro-inflammatory gut bacterial taxa and peripheral inflammation markers in cognitively impaired elderly. Neurobiol Aging. 2017;49:60-8.

9. Abraham D, Feher J, Scuderi GL, Szabo D, Dobolyi A, Cservenak M, et al. Exercise and probiotics attenuate the development of Alzheimer's disease in transgenic mice: role of microbiome. Exp Gerontol. 2019;115:122-31.

10. Bonfili L, Cecarini V, Berardi S, Scarpona S, Suchodolski JS, Nasuti C, et al. Microbiota modulation counteracts Alzheimer's disease progression influencing neuronal proteolysis and gut hormones plasma levels. Sci Rep. 2017;7:2426.

11. Liu P, Wu L, Peng G, Han Y, Tang R, Ge J, et al. Altered microbiomes distinguish Alzheimer's disease from amnestic mild cognitive impairment and health in a Chinese cohort. Brain Behav Immun. 2019;80:633-43.

12. Vogt NM, Kerby RL, Dill-McFarland KA, Harding SJ, Merluzzi AP, Johnson SC, et al. Gut microbiome alterations in Alzheimer's disease. Sci Rep. 2017;7:13537.

13. Li B, He Y, Ma J, Huang P, Du J, Cao L, et al. Mild cognitive impairment has similar alterations as Alzheimer's disease in gut microbiota. Alzheimers Dement. 2019:15:1357-66.

14. Sheng C, Lin L, Lin H, Wang X, Han Y, Liu SL. Altered gut microbiota in adults with subjective cognitive decline: the SILCODE study. J Alzheimer's Dis. 2021;82:513-26.

15. Chouraki V, Beiser A, Younkin L, Preis SR, Weinstein G, Hansson O, et al. Plasma amyloid- $\beta$ and risk of Alzheimer's disease in the Framingham Heart Study. Alzheimers Dement. 2015;11:249-57.e1.

16. Hanon O, Vidal JS, Lehmann S, Bombois S, Allinquant B, Tréluyer JM, et al. Plasma amyloid levels within the Alzheimer's process and correlations with central biomarkers. Alzheimers Dement. 2018;14:858-68.

17. Song F, Poljak A, Valenzuela M, Mayeux R, Smythe GA, Sachdev PS. Metaanalysis of plasma amyloid- $\beta$ levels in Alzheimer's disease. J Alzheimer's Dis. 2011;26:365-75.

18. Lim YY, Maruff P, Kaneko N, Doecke J, Fowler C, Villemagne VL, et al. Plasma amyloid- $\beta$ biomarker associated with cognitive decline in preclinical Alzheimer's disease. J Alzheimer's Dis. 2020;77:1057-65.

19. Nakamura A, Kaneko N, Villemagne VL, Kato T, Doecke J, Doré V, et al. High performance plasma amyloid- $\beta$ biomarkers for Alzheimer's disease. Nature. 2018:554:249-54.

20. Pérez-Grijalba V, Arbizu J, Romero J, Prieto E, Pesini P, Sarasa L, et al. Plasma A $42 / 40$ ratio alone or combined with FDG-PET can accurately predict amyloid-PET positivity: a cross-sectional analysis from the AB255 Study. Alzheimers Res Ther. 2019;1 1:96.

21. Li X, Wang X, Su L, Hu X, Han Y. Sino Longitudinal Study on Cognitive Decline (SILCODE): protocol for a Chinese longitudinal observational study to develop risk prediction models of conversion to mild cognitive impairment in individuals with subjective cognitive decline. BMJ Open. 2019:9:e028188.

22. Bondi MW, Edmonds EC, Jak AJ, Clark LR, Delano-Wood L, McDonald CR, et al. Neuropsychological criteria for mild cognitive impairment improves diagnostic precision, biomarker associations, and progression rates. J Alzheimer's Dis. 2014:42:275-89.

23. McKhann GM, Knopman DS, Chertkow H, Hyman BT, Jack CR Jr, Kawas $\mathrm{CH}$, et al. The diagnosis of dementia due to Alzheimer's disease: recommendations from the National Institute on Aging-Alzheimer's Association workgroups on diagnostic guidelines for Alzheimer's disease. Alzheimers Dement. 2011;7:263-9.

24. Zhao Q, Lv Y, Zhou Y, Hong Z, Guo Q. Short-term delayed recall of auditory verbal learning test is equivalent to long-term delayed recall for identifying amnestic mild cognitive impairment. PLoS One. 2012;7:e51157.

25. Zhao Q, Guo Q, Li F, Zhou Y, Wang B, Hong Z. The Shape Trail Test: application of a new variant of the Trail making test. PLoS One. 2013;8:e57333.

26. Guo QJL, Hong Z, Lv C. A specific phenomenon of animal fluency test in chinese elderly. Chin Mental Health J. 2007;21:622-5.

27. Guo QHZ, Shi W, Sun Y, Lv C. Boston naming test in Chinese elderly, patient with mild cognitive impairment and Alzheimer's dementia. Chin Mental Health J. 2006;20:81-4.

28. Chen $\mathrm{KL}, \mathrm{Xu} Y$, Chu AQ, Ding D, Liang XN, Nasreddine ZS, et al. Validation of the Chinese version of montreal cognitive assessment basic for screening mild cognitive impairment. J Am Geriatr Soc. 2016;64:e285-e90.

29. Qian $Y$, Yang $X, X u$ S, Wu C, Song Y, Qin N, et al. Alteration of the fecal microbiota in Chinese patients with Parkinson's disease. Brain Behav Immun. 2018;70:194-202.

30. Rognes T, Flouri T, Nichols B, Quince C, Mahé F. VSEARCH: a versatile open source tool for metagenomics. PeerJ. 2016;4:e2584.

31. Edgar RC. Search and clustering orders of magnitude faster than BLAST. Bioinformatics. 2010;26:2460-1.

32. Chen K, Roontiva A, Thiyyagura P, Lee W, Liu X, Ayutyanont N, et al. Improved power for characterizing longitudinal amyloid- $\beta$ PET changes and evaluating amyloid-modifying treatments with a cerebral white matter reference region. J Nucl Med. 2015;56:560-6.

33. LiTR, Wu Y, Jiang JJ, Lin H, Han CL, Jiang JH, et al. Radiomics analysis of magnetic resonance imaging facilitates the identification of preclinical Alzheimer's Disease: An Exploratory study. Front Cell Dev Biol. 2020:8:605734

34. DeLong ER, DeLong DM, Clarke-Pearson DL. Comparing the areas under two or more correlated receiver operating characteristic curves: a nonparametric approach. Biometrics. 1988;44:837-45.

35. Lövheim H, Elgh F, Johansson A, Zetterberg H, Blennow K, Hallmans G, et al. Plasma concentrations of free amyloid $\beta$ cannot predict the development of Alzheimer's disease. Alzheimers Dement. 2017;13:778-82.

36. Rembach A, Faux NG, Watt AD, Pertile KK, Rumble RL, Trounson BO, et al. Changes in plasma amyloid beta in a longitudinal study of aging and Alzheimer's disease. Alzheimers Dement. 2014;10:53-61.

37. Olsson B, Lautner R, Andreasson U, Öhrfelt A, Portelius E, Bjerke M, et al. CSF and blood biomarkers for the diagnosis of Alzheimer's disease: a systematic review and meta-analysis. Lancet Neurol. 2016;15:673-84.

38. Verberk IMW, Thijssen E, Koelewijn J, Mauroo K, Vanbrabant J, de Wilde A, et al. Combination of plasma amyloid beta((1-42/1-40)) and glial fibrillary acidic protein strongly associates with cerebral amyloid pathology. Alzheimers Res Ther. 2020;12:118.

39. Bhat MI, Kapila R. Dietary metabolites derived from gut microbiota: critical modulators of epigenetic changes in mammals. Nutr Rev. 2017;75:374-89.

40. Kumar H, Lund R, Laiho A, Lundelin K, Ley RE, Isolauri E, et al. Gut microbiota as an epigenetic regulator: pilot study based on whole-genome methylation analysis. mBio. 2014;5:e2113-14.

41. Megur A, Baltriukienè D, Bukelskienè V, Burokas A. The microbiota-gutbrain axis and Alzheimer's disease: neuroinflammation is to blame? Nutrients. 2020;13:37.

42. Hooper LV, Littman DR, Macpherson AJ. Interactions between the microbiota and the immune system. Science. 2012;336:1268-73.

43. Goyal D, Ali SA, Singh RK. Emerging role of gut microbiota in modulation of neuroinflammation and neurodegeneration with emphasis on Alzheimer's disease. Prog Neuropsychopharmacol Biol Psychiatry. 2021;106:110112.

44. Welcome MO. Gut microbiota disorder, gut epithelial and blood-brain barrier dysfunctions in etiopathogenesis of dementia: molecular mechanisms and signaling pathways. Neuromolecular Med. 2019;21:205-26.

45. Zhang L, Wang Y, Xiayu X, Shi C, Chen W, Song N, et al. Altered gut microbiota in a mouse model of Alzheimer's disease. J Alzheimer's Dis. 2017:60:1241-57.

46. Canfora EE, Jocken JW, Blaak EE. Short-chain fatty acids in control of body weight and insulin sensitivity. Nat Rev Endocrinol. 2015;11:577-91. 
47. Morrison DJ, Preston T. Formation of short chain fatty acids by the gut microbiota and their impact on human metabolism. Gut Microbes. 2016;7:189-200

48. Sanna S, van Zuydam NR, Mahajan A, Kurilshikov A, Vich Vila A, Võsa U, et al. Causal relationships among the gut microbiome, short-chain fatty acids and metabolic diseases. Nat Genet. 2019;51:600-5.

49. Silva YP, Bernardi A, Frozza RL. The role of short-chain fatty acids from gut microbiota in gut-brain communication. Front Endocrinol (Lausanne). 2020;11:25.

50. Braniste V, Al-Asmakh M, Kowal C, Anuar F, Abbaspour A, Tóth M, et al. The gut microbiota influences blood-brain barrier permeability in mice. Sci Transl Med. 2014;6:263ra158

51. Allin KH, Tremaroli V, Caesar R, Jensen BAH, Damgaard MTF, Bahl Ml, et al. Aberrant intestinal microbiota in individuals with prediabetes. Diabetologia. 2018;61:810-20.

52. Zhuang ZQ, Shen LL, Li WW, Fu X, Zeng F, Gui L, et al. Gut Microbiota is altered in patients with Alzheimer's disease. J Alzheimer's Dis. 2018:63:1337-46.

53. Rajilić-Stojanović M, de Vos WM. The first 1000 cultured species of the human gastrointestinal microbiota. FEMS Microbiol Rev. 2014;38:996-1047.

54. Zhan X, Stamova B, Sharp FR. Lipopolysaccharide associates with amyloid plaques, neurons and oligodendrocytes in Alzheimer's disease brain: a review. Front Aging Neurosci. 2018;10:42

55. Lukiw WJ. Bacteroides fragilis lipopolysaccharide and inflammatory signaling in Alzheimer's disease. Front Microbiol. 2016;7:1544.

56. Waite DW, Chuvochina M, Pelikan C, Parks DH, Yilmaz P, Wagner M, et al. Proposal to reclassify the proteobacterial classes Deltaproteobacteria and Oligoflexia, and the phylum Thermodesulfobacteria into four phyla reflecting major functional capabilities. Int I Syst Evol Microbiol. 2020;70:5972-6016.

57. Attene-Ramos MS, Wagner ED, Plewa MJ, Gaskins HR. Evidence that hydrogen sulfide is a genotoxic agent. Mol Cancer Res. 2006;4:9-14.

58. Sheng C, Sun Y, Wang M, Wang X, Liu Y, Pang D, et al. Combining visual rating scales for medial temporal lobe atrophy and posterior atrophy to identify amnestic mild cognitive impairment from cognitively normal older adults: evidence based on two cohorts. J Alzheimer's Dis. 2020;77:323-37.

59. Harper L, Fumagalli GG, Barkhof F, Scheltens P, O'Brien JT, Bouwman F, et al. MRI visual rating scales in the diagnosis of dementia: evaluation in 184 post-mortem confirmed cases. Brain. 2016;139:1211-25.

60. Liu YX, Qin Y, Chen T, Lu M, Qian X, Guo X, et al. A practical guide to amplicon and metagenomic analysis of microbiome data. Protein Cell. 2021;12:315-30

\section{Publisher's Note}

Springer Nature remains neutral with regard to jurisdictional claims in published maps and institutional affiliations.

Ready to submit your research? Choose BMC and benefit from:

- fast, convenient online submission

- thorough peer review by experienced researchers in your field

- rapid publication on acceptance

- support for research data, including large and complex data types

- gold Open Access which fosters wider collaboration and increased citations

- maximum visibility for your research: over $100 \mathrm{M}$ website views per year

At BMC, research is always in progress.

Learn more biomedcentral.com/submissions 